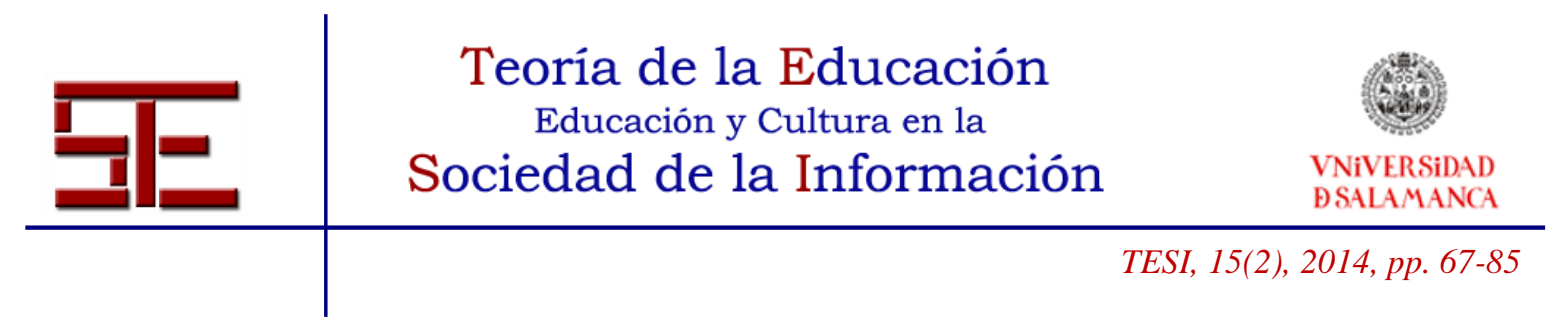

\title{
COMPARACIÓN DE TRES METODOLOGÍAS DE EVALUACIÓN DE OBJETOS DE APRENDIZAJE VIRTUALES
}

Resumen: En el presente artículo se pretende ejemplarizar la evaluación de un Objeto de Aprendizaje titulado "Construir la Geometría" usando tres propuestas diferentes que producen resultados semejantes para el objeto evaluado. Se hace una descripción de dos caminos para realizar una evaluación, la interna y externa; se utiliza inicialmente la propuesta de evaluación del grupo de investigación formado por los doctores Javier Sanz Rodríguez (Universidad Carlos III de Madrid), Juan Manuel Dodero (Universidad de Cádiz), Salvador Sánchez-Alonso (Universidad de Alcalá de Henares) y Marcela Cristina Chiarani (Universidad Nacional de San Luis-Argentina); luego la metodología desarrollada por Erla Morales Morgado en la realización de su tesis doctoral y el instrumento de evaluación usado en la Universidad Autónoma de Aguascalientes (México).

Palabras clave: Objeto de aprendizaje; instrumento; evaluación.

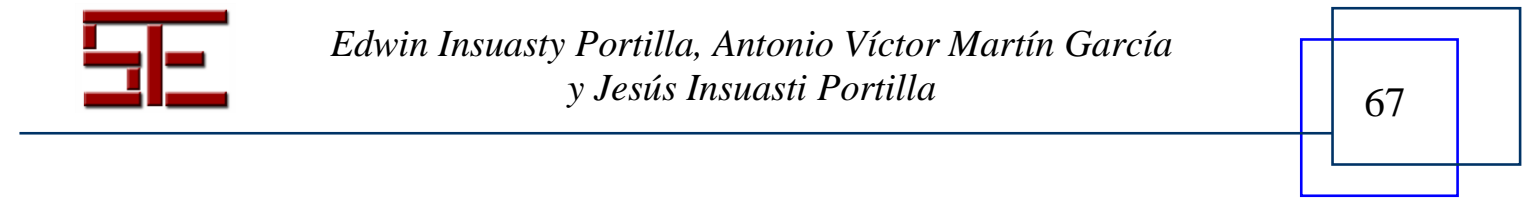




\title{
COMPARISON OF THREE ASSESSMENT METHODOLOGIES OF VIRTUAL LEARNING OBJECTS
}

\begin{abstract}
This article is intended to exemplify the evaluation of a learning object titled "Building Geometry" using three different proposals that produce similar results for the object being evaluated. A description of two paths is made for an evaluation, internal and external; the proposal evaluation of research group formed by Drs Javier Sanz Rodríguez (Carlos III de Madrid University), Juan Manuel Dodero (University of Cádiz), Salvador Sanchez-Alonso (University of Alcalá de Henares) and Marcela Cristina Chiarani (National University of San Luis-Argentina) is initially used; then the methodology developed by Erla Morales Morgado in his doctoral thesis and the third proposal is the assessment tool used in the Autonomous University of Aguascalientes (Mexico).
\end{abstract}

Key words: Learning object; instrument; evaluation. 


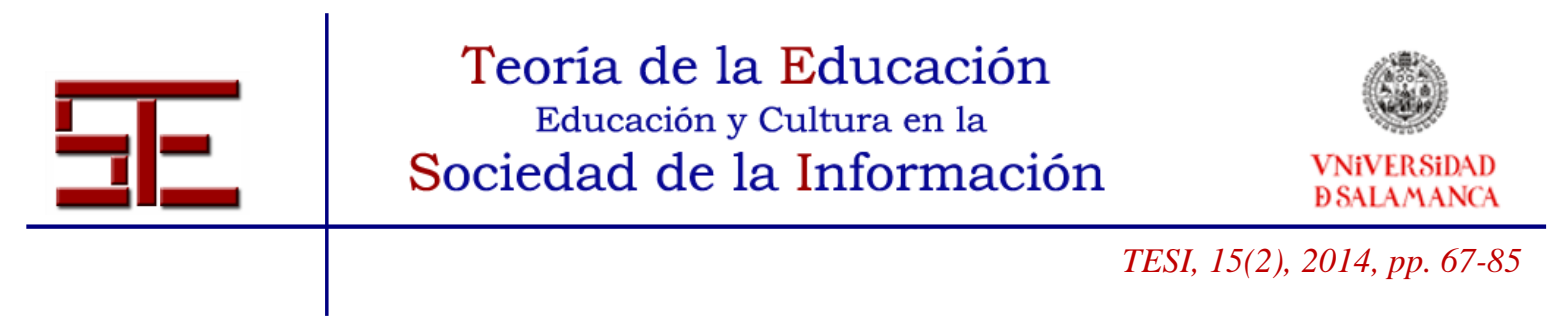

\section{COMPARACIÓN DE TRES METODOLOGÍAS DE EVALUACIÓN DE OBJETOS DE APRENDIZAJE VIRTUALES}

Fecha de recepción: 01/02/2014; fecha de aceptación: 07/04/2014; fecha de publicación: 30/06/2014

Edwin Insuasty Portilla

edwinsuasty@gmail.com

Universidad de Nariño

Antonio Víctor Martín García

avmg@usal.es

Universidad de Salamanca

Jesús Insuasti Portilla

insuasty@udenar.edu.co

Universidad de Nariño

\section{1.- INTRODUCCIÓN}

Es común encontrar definiciones, estrategias, técnicas y métodos para la evaluación de los procesos de aprendizaje y en general sobre la calidad de la educación, pero menos común aquellos que se relacionan con la evaluación de los materiales educativos empleados en el proceso. También se conocen diversas metodologías para la evaluación de software, en particular para el software educativo, pero pocas para una clase especial conocida como los Objetos de Aprendizaje.

La evaluación de Objetos de Aprendizaje, en el fondo, debe medir la calidad del objeto en el sentido educativo independientemente de la clase de tecnología empleada. Se encuentran disponibles en Internet grandes cantidades de Objetos de Aprendizaje, muchos de ellos a los cuales no se les ha medido su calidad y eficiencia, lo que aumenta las críticas a la utilización de estos recursos en el aprendizaje. Muchos de estos objetos no están en repositorios que tienen criterios de calidad o sistemas de evaluación y no hay mecanismo conocido para que algunos Objetos de Aprendizaje de mala calidad sean eliminados de la Red o delimitar su acceso.

Northrup (2007) advierte de? ciertas dificultades que se presentan en la evaluación de Objetos de Aprendizaje debido a la creciente cantidad de ellos, para lo cual cita como ejemplo las evaluaciones en el Repositorio MERLOT:

La evaluación de objetos de aprendizaje es una tarea difícil, pero hay que tenerla en cuenta si la expectativa es de uso a gran escala. El Repositorio MERLOT es un sistema de revisión por pares que

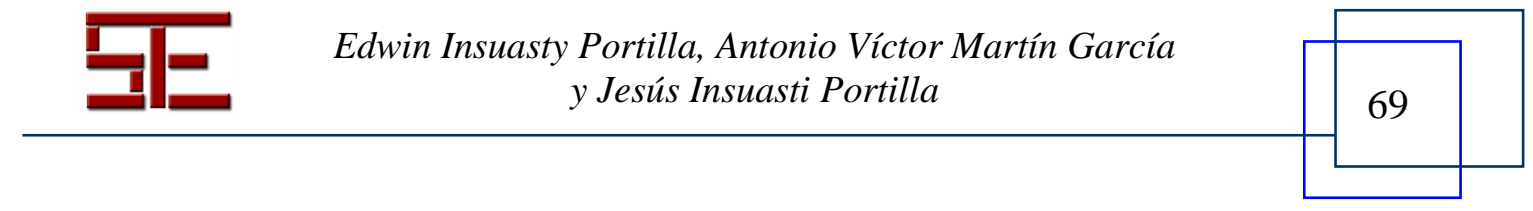




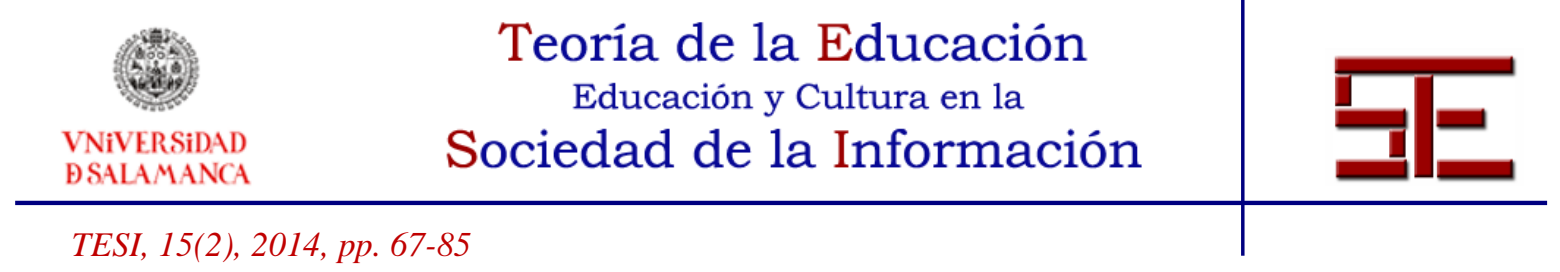

requiere que todos los objetos sean evaluados antes de publicarlos. Esto a veces causa un cuello de botella enorme para que objetos nuevos puedan estar disponibles al mismo tiempo en que las comunidades no cesan de enviar objetos. Sin embargo, los objetos revisados por pares ofrecen mucho mérito a la comunidad académica en su conjunto (Northrup, 2007).

\section{1.- Definición de Objeto de Aprendizaje}

Desde los años 60 se empezó a utilizar entre los programadores de computadores lo que se conoce como programación orientada a objetos, concepto que se trató de llevar al ámbito educativo mediante diferentes tipos de materiales diseñados bajo nuevas concepciones basadas en las ideas que fundamentan el paradigma de programación mencionado.

Estas ideas fueron tomadas para la formulación de lo que son los Objetos de Aprendizaje (OA). "El término Objetos de Aprendizaje fue popularizado por Wayne Hodgins en 1994 cuando nombró la CedMA (Computer Education Management Association) trabajando en el grupo Learning Architectures, APIs and Learning Objects'. CEdMA, describe sus propios propósitos como una provisión de un foro de discusión de cuestiones en entrenamiento por computador"' (Friesen, 2004).

Se usa una metáfora para describir lo que se conoce como Repositorio de Objetos de Aprendizaje: "Un repositorio de objetos de aprendizaje es como un diccionario, un conjunto organizado de palabras que se pueden combinar fácilmente y volver a combinarse en múltiples y significativas frases" (Acker; Pearl et al., 2003), en cambio, los escépticos de esta nueva concepción de materiales educativos opinan que "los Objetos de Aprendizaje son simplemente un término de moda para hacer referencia a materiales, 'repositorio' como una biblioteca, 'metadatos' como un registro de un catálogo y 'secuencia estructurada' como un plan de estudios. Ellos concluyen que el conocimiento es una experiencia subjetiva en una comunidad de aprendices y no un objeto que puede transportarse hasta sus disciplinas" (ibid.).

Muchas definiciones de Objeto de Aprendizaje se han formulado, entre las que se cuentan la del profesor David A. Wiley II de la Utah State University:

Los Objetos de Aprendizaje son elementos de un nuevo tipo de instrucción basada en computadores enmarcada en el paradigma orientado a objetos de las ciencias de la computación. La orientación a objetos valora altamente la creación de componentes (llamados objetos) que pueden ser reutilizados en múltiples contextos. Esta es la idea fundamental detrás de los Objetos de Aprendizaje: diseños instruccionales que pueden construirse en pequeños componentes instruccionales (comparados con el tamaño de un curso completo), que pueden ser reutilizados un número de veces en diferentes contextos de aprendizaje. Adicionalmente, los Objetos de Aprendizaje son generalmente entendidos como entidades digitales para ser entregadas por Internet, significando que cualquier número de personas pueden acceder a ellos y usarlos simultáneamente (como oposición a los tradicionales medios de instrucción tal como un dispositivo de reproducción de video que solo puede existir en un lugar a la vez). Por otra parte, aquellos que incorporan objetos de aprendizaje pueden colaborar y beneficiarse inmediatamente de las nuevas versiones. Estas diferencias significativas entre los objetos de aprendizaje y otros medios de instrucción han existido anteriormente (Wiley, 2002).

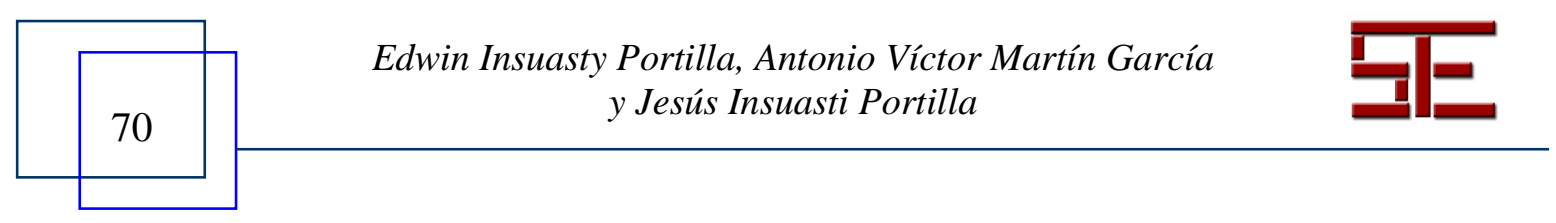




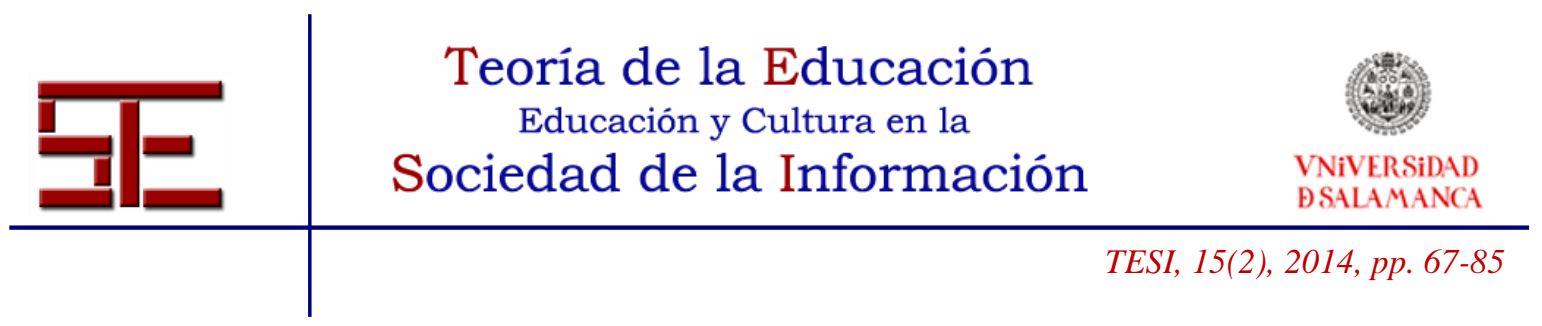

Otra definición es la de Morales y García et al. (2005), quienes afirman que un OA es: "una unidad con un objetivo de aprendizaje, caracterizada por ser digital, independiente, con una o pocas ideas relacionadas y accesible a través de metadatos con la finalidad de ser reutilizadas en diferentes contextos y plataformas" (Morales; García et al., 2005). Según Wiley (2003), "un Objeto de Aprendizaje es cualquier recurso digital que puede ser reutilizado para mediar en el aprendizaje (Wiley; Edwards, 2002). Las dos principales metas detrás de la investigación y desarrollo de los Objetos de Aprendizaje son:

1. Mejorar el costo de la instrucción online, y

2. Habilitar la innovación pedagógica (Wiley, 2003).

Algo similar son los llamados Objetos Instruccionales de Gibbon, Nelson y otros:

A como nosotros usamos el término en este capítulo, los Objetos Instruccionales se refieren a cualquier elemento de una arquitectura que puede ser señalado independientemente dentro de un ensamblaje momentáneo en orden a crear un evento instruccional. El Objeto Instruccional puede incluir ambientes de problemas, modelos interactivos, problemas o conjuntos de problemas instruccionales, módulos de funciones instruccionales, rutinas modulares para el incremento instruccional (entrenamiento, retroalimentación, etc.), elementos de mensajes instruccionales, rutinas modulares para la representación de información, o módulos lógicos relacionados con los propósitos instruccionales (administración, registro, selección, etc.) (Gibbons; Nelson et al., 2002).

Del análisis de las anteriores definiciones sobre el significado de un OA, se puede concluir que los Objetos de Aprendizaje son componentes individuales de instrucción en formatos digitales distribuibles mediante redes con características claramente definidas que permiten ser referenciados para ser reutilizados y ensamblados en orden a formar unidades de instrucción mayores.

\section{2.- Los metadatos}

Un OA, para ser clasificado, catalogado y posteriormente reutilizado, necesita tener definidos sus metadatos, que literalmente significa datos que describen otros datos. La metáfora más cercana son las fichas de una biblioteca, las cuales describen cada una un libro. En este caso el libro sería el OA y la ficha sus metadatos. De igual forma, para una lata de alimentos, la información contenida en su etiqueta corresponde a los metadatos. Uno de los esquemas más usados para la descripción de los metadatos de un OA es el LOM (Learning Object Metadata), que propone 9 características mínimas con 47 aspectos a considerar en ellas y que su finalidad es sintetizada así: "El propósito del desarrollo del estándar LOM es facilitar la búsqueda, evaluación, adquisición y uso de Objetos de Aprendizaje por estudiantes y profesores" (Lightle, 2003).

La siguiente tabla es un resumen de las características y aspectos del esquema LOM:

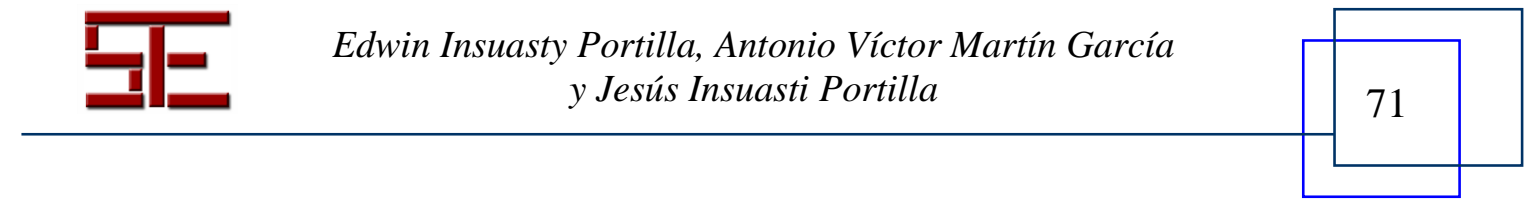




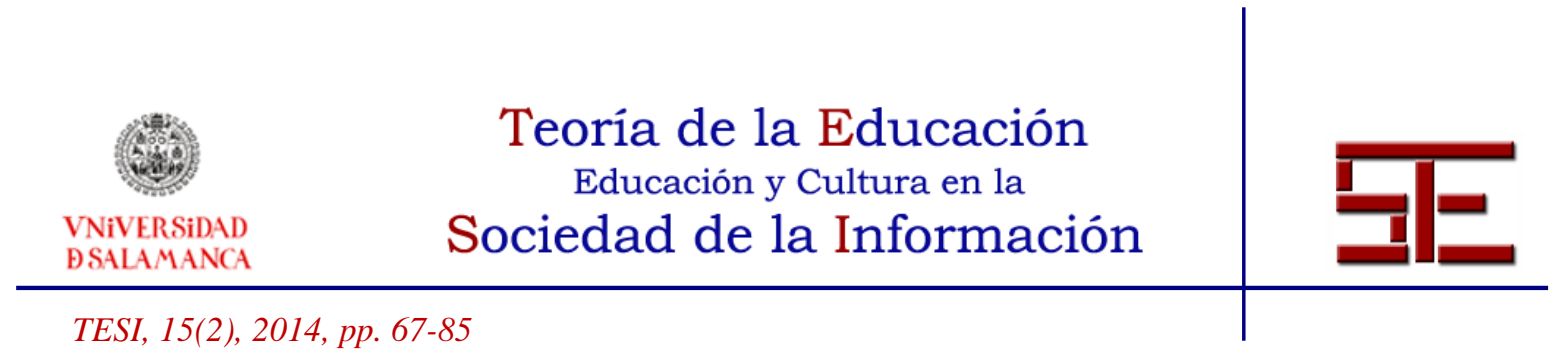

\begin{tabular}{|c|c|}
\hline Características & Aspectos \\
\hline 1. General & $\begin{array}{l}\text { Identificador, Título, Entrada de catálogo, } \\
\text { Lengua, Descripción, Descriptor, Cobertura, } \\
\text { Estructura, Nivel de agregación }\end{array}$ \\
\hline 2. Ciclo de vida & Versión, Estatus, Otros colaboradores \\
\hline 3. Metainformación & $\begin{array}{l}\text { Identificador, Entrada de catálogo, Otros } \\
\text { colaboradores, Esquema de metadatos, Lengua }\end{array}$ \\
\hline 4. Técnica & $\begin{array}{l}\text { Formato, Tamaño, Ubicación, Requisitos, } \\
\text { Comentarios sobre la instalación, Otros } \\
\text { requisitos para plataformas, Duración }\end{array}$ \\
\hline 5. Uso educativo & $\begin{array}{l}\text { Tipo de interactividad, Tipo de recurso de } \\
\text { aprendizaje, Nivel de interactividad, Densidad } \\
\text { semántica, Usuario principal, Contexto (Nivel } \\
\text { educativo), Edad, Dificultad, Tiempo previsto de } \\
\text { aprendizaje, Descripción, Lengua }\end{array}$ \\
\hline 6. Derechos & $\begin{array}{l}\text { Costo, Copyright y otras restricciones, } \\
\text { Descripción }\end{array}$ \\
\hline $\begin{array}{l}\text { 7. Relación (con otros } \\
\text { recursos) }\end{array}$ & $\begin{array}{l}\text { Tipo (naturaleza de la relación con el recurso } \\
\text { principal), Recurso (recurso principal al que se } \\
\text { refiere esta relación) }\end{array}$ \\
\hline 8. Observaciones & Persona, Fecha, Descripción \\
\hline 9. Clasificación & $\begin{array}{l}\text { Finalidad, Nivel taxonómico, Descripción, } \\
\text { Descriptor }\end{array}$ \\
\hline
\end{tabular}

De la calidad de los metadatos descriptores del OA depende también la evaluación que se haga del mismo porque ellos proveen de mucha información pedagógica del objeto. De todos modos, la evaluación siempre tiene un carácter subjetivo, siendo esta una habilidad que se adquiere en buena medida por la experiencia.

La habilidad para calificar la calidad de un objeto, vista sobre su utilidad o la provisión de detalles de cómo se integra dentro de una experiencia específica de aprendizaje debería incrementar la inmediata usabilidad y la adopción de repositorios y Objetos de Aprendizaje. La habilidad para evaluar Objetos de Aprendizaje debería ser algo que cada uno pueda hacer pero el proceso necesita ser racionalizado para que sea satisfactorio. Además, los metadatos acerca de la calidad del contenido no están adecuadamente soportados en la especificación de metadatos existente (Halm, 2003).

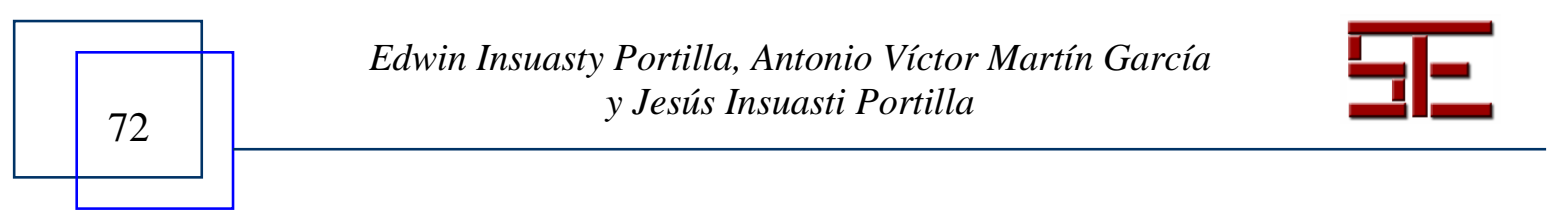




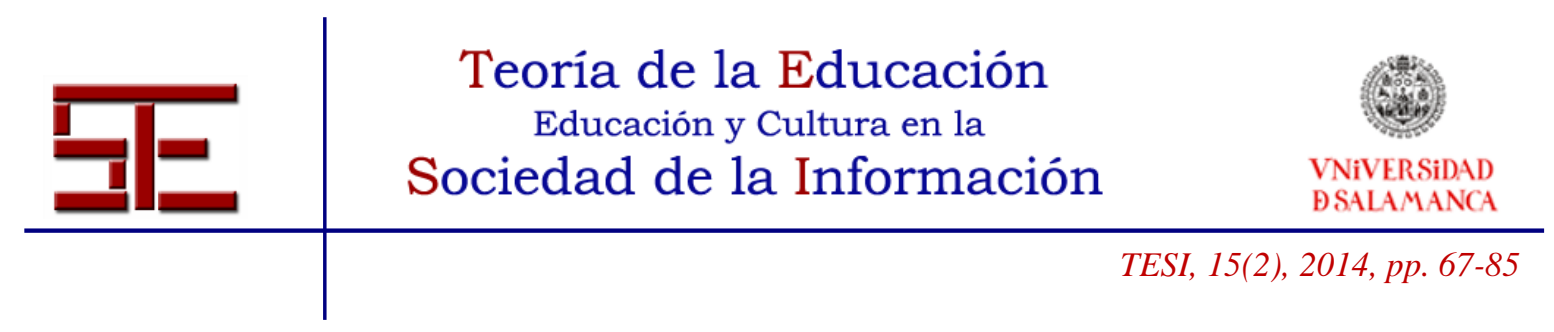

\section{2.- EVALUACIÓN DE UN OBJETO DE APRENDIZAJE}

Una técnica para evaluar Objetos de Aprendizaje es la conocida como collaborative filtering, donde se involucran múltiples agentes, puntos de vista y datos de diferentes procedencias en un ambiente colaborativo.

Esta técnica selecciona el contenido basándose en las preferencias de usuarios con intereses similares, básicamente a través de fusionar y ordenar las opiniones (o experiencias de uso) en cada área temática. En otras palabras, hay un sistema automático que recoge la información de las acciones de los usuarios (explícitas, como votar o contestar una pregunta; o implícitas, como ver qué enlaces se han visitado y cuáles no, y durante cuánto tiempo) y determina la importancia de cada contenido comparándola con el resto de información recogida a partir del uso de todos los usuarios (Ferran Ferrer; Minguillón, 2005).

La evaluación de un Objeto de Aprendizaje puede ser hecha por dos caminos, mediante un evaluador externo al equipo del proyecto que diseñó el objeto y el otro es utilizar a los usuarios del objeto como evaluadores del mismo. Williams (2002) propone una serie de pasos para adelantar una evaluación externa:

-Paso 1. Diseñar un formulario de solicitud de evaluadores donde se indique lo que el equipo de desarrollo del objeto pretende; los recursos que estarían a disposición como personal, tiempo, fondos, etc., e invitarlos a hacer una propuesta con la posibilidad de que hagan preguntas y así exploren la situación en persona.

-Paso 2. Después de recibir las diferentes propuestas de profesionales interesados en la evaluación del objeto, con sus diferentes filosofías de evaluación, sus experiencias, recursos e intereses, se debe hacer la selección del evaluador enfocándolo hacia el objetivo del proyecto.

- Paso 3. Especificar un contrato para el equipo de evaluación externo, basado en la propuesta de evaluación presentada por él o ellos. El contrato debe especificar las responsabilidades de ambas partes, así como el marco referencial y los informes que se han de elaborar de acuerdo a un calendario.

- Paso 4. Mantener unas reuniones iniciales entre las dos partes y con otras personas que influirían en el proyecto. Estas entrevistas aclararían aspectos como qué usuarios potenciales se buscan, sus definiciones de la evaluación, valores y criterios que se podrían utilizar y lo que se podría hacer con diferentes tipos de resultados.

-Paso 5. El equipo evaluador hace una revisión de su propuesta con base en las entrevistas que le han permitido descubrir conflictos de criterios o valores entre los diferentes grupos.

-Paso 6. Una vez que se han clarificado intereses, el equipo de evaluación debe sintetizar los aspectos esenciales de la evaluación que se abordarán durante su estudio. Deben perfeccionar la recopilación de datos y análisis de los planes de la propuesta original para orientar el estudio a la luz de los recursos disponibles. Por último, se

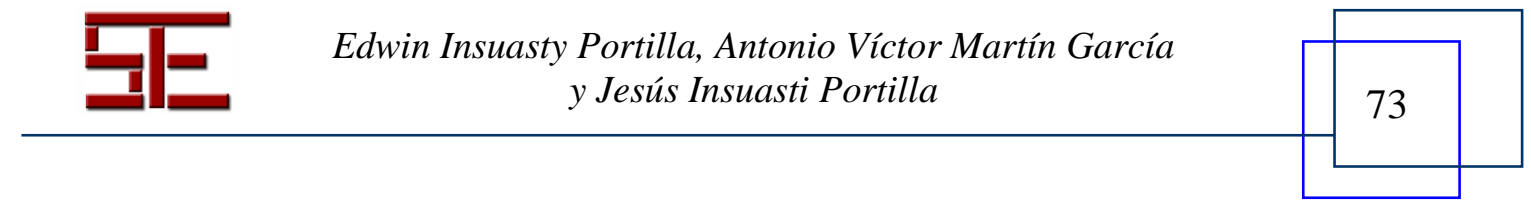




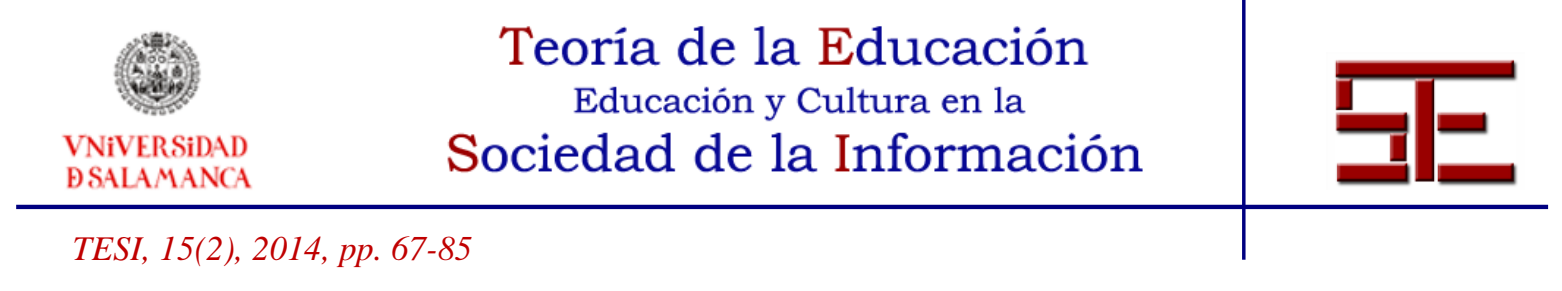

llevará a cabo el estudio de acuerdo con estos planes y la entrega del informe de los resultados a los participantes interesados o sus representantes.

-Paso 7. Finalmente, todo el proceso usado para la evaluación debería ser metaevaluado para asegurarse de que está acorde con los estándares establecidos con los diseñadores del objeto y los proponentes de la evaluación (Williams, 2002).

Para el caso de la evaluación interna, se recomiendan tres pasos descritos como sigue:

-Paso 1. Involucrar en la fase del diseño instruccional a los potenciales usuarios a quienes va dirigido el objeto o a representantes de ellos para contextualizar tanto la evaluación del objeto como la evaluación del proceso de aprendizaje.

-Paso 2. Establecer un diálogo sistemático y constante con todos los involucrados en el proceso para recoger sus opiniones en cada etapa del proceso de diseño del objeto.

-Paso 3. Metaevaluar tanto interna como externamente. El equipo de diseño necesita una constante retroalimentación provista por las personas que se incorporaron (estudiantes, otros profesores). Estos deben designar a una persona que está continuamente revisando el proceso, criticándolo y observando de cerca los resultados obtenidos. Los informes periódicos a los miembros del equipo acerca de lo bien que se están cumpliendo las normas de evaluación no sólo mejorarán la evaluación de los objetos de aprendizaje, sino que mejorarán el aprendizaje (ibid.).

MERLOT (Multimedia Educational Resource for Learning and Online Teaching) es un repositorio de Objetos de Aprendizaje desarrollado por colaboración de diferentes estudiantes de nivel superior y profesores de diferentes facultades. El sistema de evaluación de MERLOT es externo, ya que se hace a través de pares, después de que el objeto haya sido incorporado a su base de datos.

Una vez que un recurso es asignado a un evaluador, aparece en MERLOT como "bajo revisión". Esta evaluación es realizada por al menos dos personas altamente competentes, quienes desde una perspectiva individual crean una "revisión compuesta". Este material permanece catalogado como "bajo revisión" hasta que el resultado de la revisión por pares es publicado en MERLOT (Morales Morgado, 2007).

Northrup propone una serie de preguntas iniciales ante la evaluación de un OA:

Las preguntas de evaluación pueden incluir: (1) ¿Concuerdan las metas y los objetivos? (2) ¿Va a encajar en el contexto de la lección o el curso? (3) ¿Es de alta calidad? (4) ¿Es preciso y libre de prejuicios? (5) ¿Se puede utilizar por mis alumnos, son plug-ins o se necesita software adicional para funcionar? (6) ¿Es el tamaño del archivo demasiado grande para descargar? (7) ¿Cómo descargo el objeto o cómo enlazarlo? (8) ¿Tengo confianza en que seguirá situado en su enlace designado? (Northrup, 2007).

Sobre los aspectos de la calidad, se puede considerar la siguiente tabla de evaluación propuesta por Vidal, Segura et al. (2008):

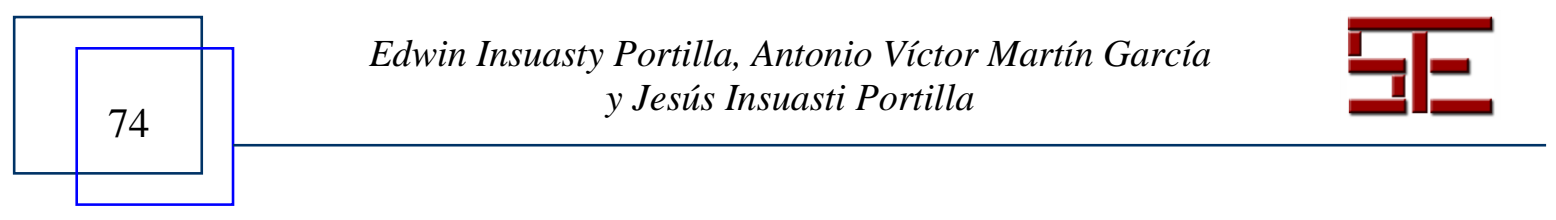




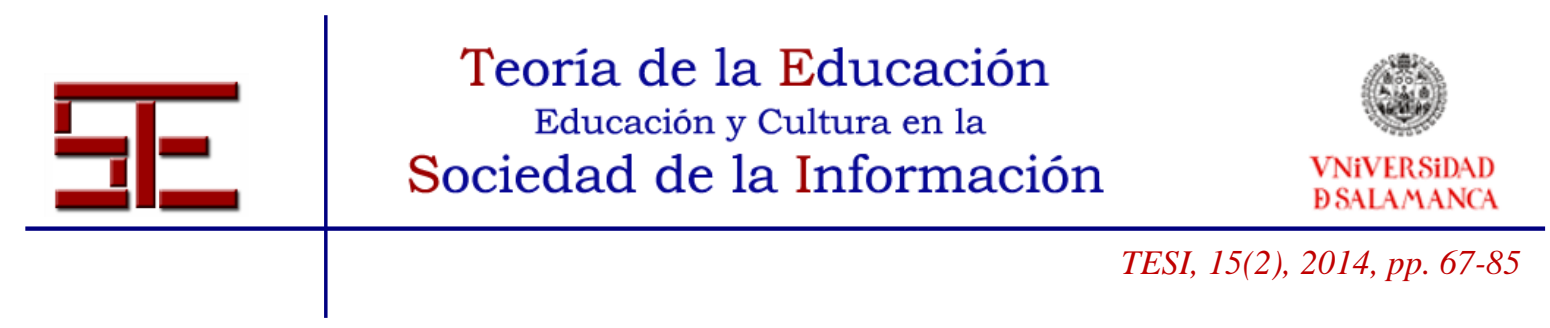

\begin{tabular}{|l|l|}
\hline ATENCIÓN & ASPECTOS DE LA CALIDAD \\
\hline \multirow{2}{*}{ Producto } & Calidad en contenido y estructura interna y uso del OA \\
\cline { 2 - 2 } & Calidad en potencial pedagógico \\
\cline { 2 - 2 } & Calidad en metadatos \\
\hline Proceso & $\begin{array}{l}\text { Calidad en cuanto a los procedimientos, métodos o } \\
\text { técnicas utilizadas en el desarrollo }\end{array}$ \\
\cline { 2 - 2 } & $\begin{array}{l}\text { Calidad en los productos de trabajo intermedio, y el OA } \\
\text { como producto final de la última etapa de desarrollo }\end{array}$ \\
\hline
\end{tabular}

Otro aspecto importante en la evaluación de Objetos de Aprendizaje es la reusabilidad. Aunque esta se puede estudiar a través del análisis de los metadatos del objeto, se deben tener algunos criterios para entrar a evaluarla. Sanz Rodríguez, Dodero et al. (2008) proponen una serie de aspectos que determinan la reusabilidad de un Objeto de Aprendizaje:

a) Desde el punto de vista estructural:

Autocontenido. Debe tener sentido por sí mismo. Las referencias a otros recursos pueden disminuir su reusabilidad y la presencia de prerrequisitos necesarios para ser realizado puede dificultar su adaptación a otros contextos.

Modular. Para poder ser combinado con otros objetos formando lecciones o cursos.

De una granularidad adecuada. Un tamaño adecuado y un objetivo educativo específico facilitarán su reutilización.

Localizable. El objeto debe ser identificable a través de sus metadatos, que deben estar correctamente rellenados.

Modificable. El objeto debe tener la capacidad de ser modificado para poder reformularlo adaptándolo a un determinado contexto, aumentando así las posibilidades de reutilización. La complejidad de comprensión de un objeto de aprendizaje puede dificultar su reformulación para poder reutilizarlo en una unidad de aprendizaje.

Fácil de usar y con una interfaz intuitiva.

Estandarizado. Diseñado siguiendo unos estándares adecuados.

b) Desde el punto de vista tecnológico:

Dependencias de plataformas y de software necesario para acceder a los objetos.

Dependencias de representación. Los objetos deberían separar los contenidos del formato de representación.

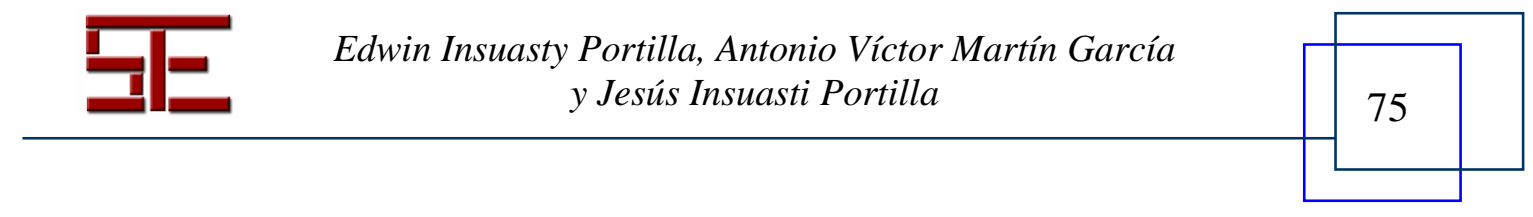




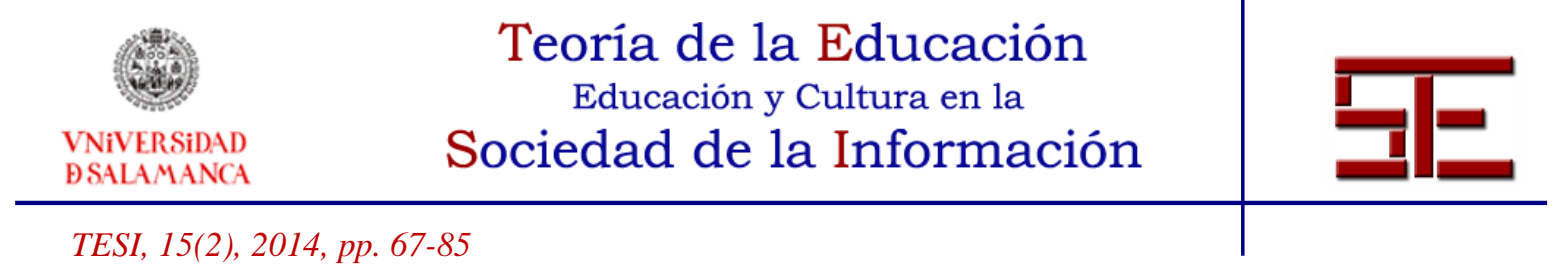

c) Desde el punto de vista educativo:

Genericidad. Cuanto más genérico sea, más reutilizable será. Los objetos de aprendizaje que traten aspectos más generales serán más fácilmente reutilizables que aquellos que sean más avanzados o especializados.

Preparado para ser usado en diferentes niveles educativos, con diferentes contenidos y evaluaciones.

Pedagógicamente neutral. Utilizable con cualquier metodología educativa.

Carente de dependencias institucionales, legales, sociales y culturales.

Independiente de la ubicación desde donde se cursa.

Independiente del tiempo. No depende de la ocurrencia de determinados eventos (Sanz Rodríguez; Dodero et al., 2008).

\section{3.- COMPARACIÓN DE TRES METODOLOGÍAS DE EVALUACIÓN}

El Objeto de Aprendizaje que se ha escogido para su evaluación se titula "Construir la Geometría”, publicado por la Junta de Andalucía, Consejería de Educación y Ciencia en el sitio:

http://www.juntadeandalucia.es/averroes/html/adjuntos/2007/09/11/0012/indexflash.htm

Este objeto de aprendizaje permite abordar muchos aspectos de la geometría plana donde los alumnos pueden explorar los conceptos interactivamente.

Basados en la tabla de evaluación de Sanz Rodríguez, Dodero et al., tenemos los siguientes resultados:

Ejemplo de la evaluación de la reusabilidad del Objeto "Construir la Geometría"

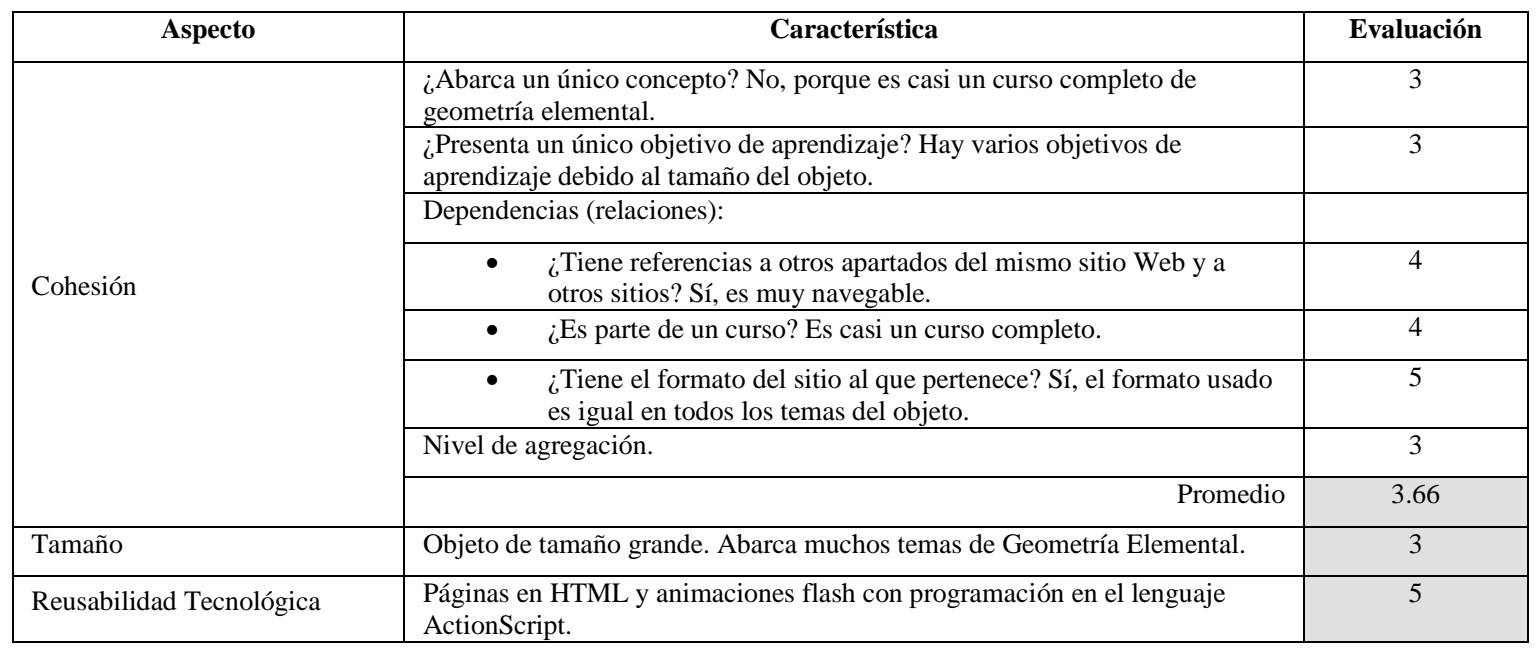

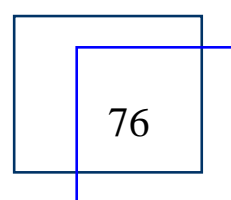

Edwin Insuasty Portilla, Antonio Víctor Martín García

y Jesús Insuasti Portilla 


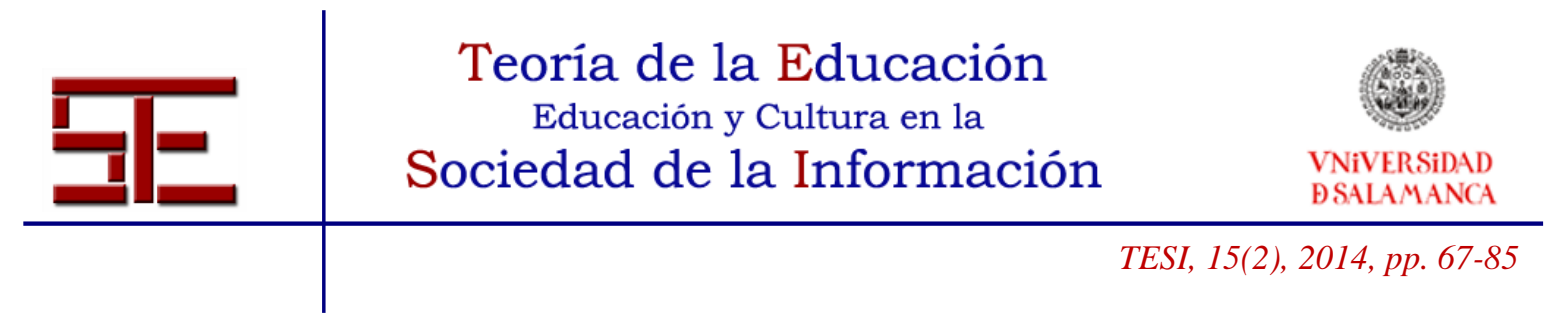

\begin{tabular}{|l|l|c|}
\hline \multirow{5}{*}{ Reusabilidad Educativa } & Contexto: Escuela Primaria. & 4 \\
\cline { 2 - 4 } & Lenguaje: Español. & 4 \\
\cline { 2 - 4 } & Rango de Edades: Primaria y Secundaria. & 4 \\
\cline { 2 - 4 } & Clasificación: Ciencia->Matemáticas->Geometría Euclidiana. & 4 \\
\cline { 2 - 3 } & & 4.0 \\
\hline
\end{tabular}

Tabla de Evaluación en (Sanz Rodríguez; Dodero et al., 2008).

El promedio de los cuatro aspectos evaluados es 3.915.

Morales Morgado (2007) propone unos criterios de evaluación para Objetos de Aprendizaje, clasificados como:

1. Criterios pedagógicos

a) Categoría psicopedagógica

b) Categoría didáctico-curricular

2. Criterios de usabilidad

a) Categoría diseño de interfaz

b) Categoría diseño de navegación

Se utilizará el instrumento propuesto por esta autora para evaluar el objeto "Construir la Geometría".

\section{Criterios sobre Aspectos Pedagógicos (Categoría Psicopedagógica)}

\begin{tabular}{|l|c|}
\hline CRITERIOS PEDAGÓGICOS PARA EVALUAR OBJETOS DE APRENDIZAJE & $\begin{array}{c}\text { N/S No Sabe, } 1 \text { Muy } \\
\text { Deficiente, } 2 \text { Deficiente, } \\
\text { 3 Aceptable, } \\
\text { 4 Alta, } 5 \text { Muy Alta }\end{array}$ \\
\hline CATEGORÍA PSICOPEDAGÓGICA & 4 \\
\hline MOTIVACIÓN Y ATENCIÓN & 3 \\
\hline Presentación atractiva y original: captar la atención de los estudiantes y mantener el interés. & \\
\hline $\begin{array}{l}\text { Información relevante: entregar información importante para ayudar a comprender los } \\
\text { contenidos. }\end{array}$ & 4 \\
\hline Participación del alumno: explicar claramente su participación en el desarrollo del programa. & \\
\hline DESEMPEÑO PROFESIONAL & 4 \\
\hline $\begin{array}{l}\text { Adecuación a competencias profesionales: adecuar la utilidad de los contenidos y actividades a } \\
\text { las necesidades y desempeño profesional de los estudiantes. }\end{array}$ & \\
\hline NIVEL DE DIFICULTAD ADECUADO A LAS CARACTERÍSTICAS DE LOS ESTUDIANTES & 5 \\
\hline $\begin{array}{l}\text { Profundidad pertinente: adecuar profundidad según conocimientos previos y nivel de } \\
\text { complejidad que el estudiante es capaz de comprender. }\end{array}$ & \\
\hline $\begin{array}{l}\text { Nivel de lenguaje: adecuar lenguaje utilizado (científico, etc.) a los conocimientos previos de los } \\
\text { estudiantes. }\end{array}$ & \\
\hline INTERACTIVIDAD & 4 \\
\hline
\end{tabular}

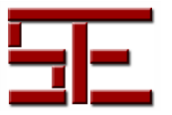

Edwin Insuasty Portilla, Antonio Víctor Martín García y Jesús Insuasti Portilla 


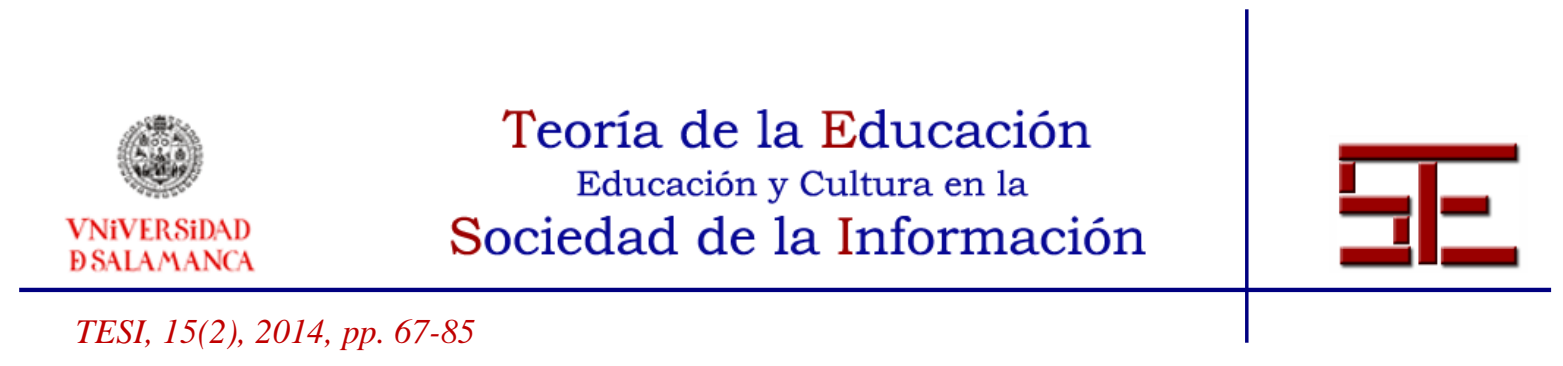

\begin{tabular}{|l|c|}
\hline $\begin{array}{l}\text { Nivel de interactividad: promover actividades abiertas, diversas maneras de resolver problemas, } \\
\text { proporcionar realimentación y corrección de errores. }\end{array}$ & 5 \\
\hline $\begin{array}{l}\text { Tipo de interactividad: adecuar interactividad a los objetivos de la metodología, los niveles } \\
\text { pueden ser: activos, expositivos o mixtos. }\end{array}$ & 4 \\
\hline CREATIVIDAD & \\
\hline Promover el desarrollo e iniciativa y el aprendizaje autónomo. & 3 \\
\hline $\begin{array}{l}\text { Promover el desarrollo de habilidades metacognitivas y estrategias de aprendizaje que les } \\
\text { permitan planificar, regular y evaluar su propia actividad intelectual. }\end{array}$ & 2 \\
\hline PUNTUACIÓN FINAL & 3.80 \\
\hline \multicolumn{1}{|c|}{ COMENTARIOS GENERALES (Si considera que el objeto puede ser reutilizado en otras áreas, dé algunos ejemplos) } \\
\hline
\end{tabular}

\section{Criterios sobre Aspectos Pedagógicos (Categoría Didáctico-Curricular)}

\begin{tabular}{|c|c|}
\hline CRITERIOS PEDAGÓGICOS PARA EVALUAR OBJETOS DE APRENDIZAJE & $\begin{array}{c}\text { N/S No Sabe, } 1 \text { Muy } \\
\text { Deficiente, } 2 \text { Deficiente, } \\
3 \text { Aceptable, } \\
4 \text { Alta } 5 \text { Muy Alta }\end{array}$ \\
\hline \multicolumn{2}{|l|}{ CATEGORÍA DIDÁCTICO-CURRICULAR } \\
\hline \multicolumn{2}{|l|}{ CONTEXTO } \\
\hline Nivel formativo adecuado a la situación educativa, por ejemplo: educación secundaria, etc. & 5 \\
\hline Unidad. & 4 \\
\hline \multicolumn{2}{|l|}{ OBJETIVOS } \\
\hline $\begin{array}{l}\text { Correctamente formulado: generalmente los objetivos se elaboran según la fórmula: verbo } \\
\text { infinitivo + contenido + }\end{array}$ & 2 \\
\hline Factible: puede ser alcanzado. & 4 \\
\hline $\begin{array}{l}\text { Indica lo que se espera que sea aprendido: el alumno debe ser consciente de lo que tiene que } \\
\text { aprender. }\end{array}$ & 4 \\
\hline $\begin{array}{l}\text { Coherente con los objetivos generales: los objetivos específicos deben ayudar a cumplir los } \\
\text { objetivos generales. }\end{array}$ & 4 \\
\hline \multicolumn{2}{|l|}{ TIEMPO DE APRENDIZAJE } \\
\hline Presentar información suficiente y adecuada al nivel educativo. & 4 \\
\hline Adecuar los contenidos al objetivo propuesto. & 4 \\
\hline Presentar información en distintos formatos (texto, audio, etc.). & 4 \\
\hline Permite interactuar con el contenido a través de enlaces. & 5 \\
\hline $\begin{array}{l}\text { Presentar información complementaria para ayudar a los alumnos que deseen profundizar sus } \\
\text { conocimientos. }\end{array}$ & 2 \\
\hline $\begin{array}{l}\text { Cuidar que la información que presenta sea confiable (datos exactos, referencias bibliográficas, } \\
\text { etc.). }\end{array}$ & 4 \\
\hline $\begin{array}{l}\text { Presentar la información de forma adecuada para ayudar a una mejor comprensión del } \\
\text { contenido. }\end{array}$ & 4 \\
\hline
\end{tabular}

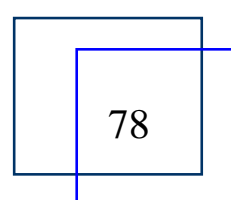

Edwin Insuasty Portilla, Antonio Víctor Martín García y Jesús Insuasti Portilla 


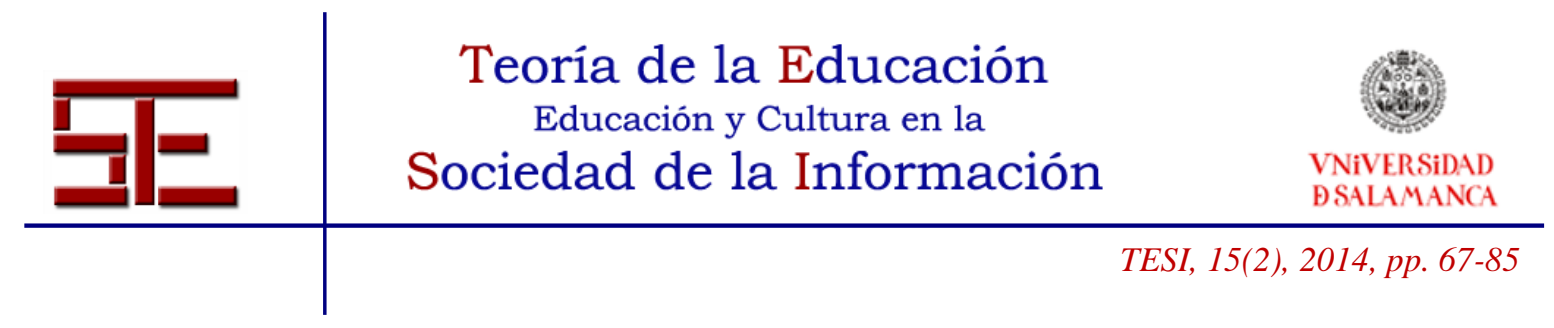

\begin{tabular}{|l|c|}
\hline Verificar que el idioma empleado en los contenidos sea pertinente a los objetivos de enseñanza. & 5 \\
\hline ACTIVIDADES & 4 \\
\hline $\begin{array}{l}\text { Ayuda a reforzar los conceptos. } \\
\text { Promueve una participación activa: estimulala reflexión y la crítica, esto es, el cuestionamiento } \\
\text { de las propias ideas para la integración de la nueva información a los conocimientos } \\
\text { preexistentes. }\end{array}$ & 4 \\
\hline $\begin{array}{l}\text { Presenta distintos tipos de estrategias de aprendizaje, según sea el caso (resolución de } \\
\text { problemas, estudio de caso). }\end{array}$ & 4 \\
\hline \begin{tabular}{l} 
Presenta actividades de evaluación y práctica. \\
\hline Se propone modalidad de trabajo según sea el caso (individual, colaborativa y/o cooperativa).
\end{tabular} & 5 \\
\hline REALIMENTACIÓN & 3 \\
\hline Se refuerzan los conocimientos a través de ejercicios, autoevaluaciones, etc. & 2 \\
\hline PUNTUACIÓN FINAL & 3.85 \\
\hline \multicolumn{1}{|c|}{ COMENARIOS GENERALES (Si considera que el objeto puede ser reutilizado en otras áreas, dé algunos ejemplos) } \\
\hline
\end{tabular}

\section{Criterios de Usabilidad (Diseño de Interfaz)}

\begin{tabular}{|l|c|}
\hline CRITERIOS DE USABILIDAD PARA EVALUAR OBJETOS DE APRENDIZAJE & $\begin{array}{c}\text { N/S No Sabe, } 1 \text { Muy } \\
\text { Deficiente, 2 Deficiente, } \\
\text { 3 Aceptable, } \\
\text { 4 Alta 5 Muy Alta }\end{array}$ \\
\hline DISEÑO DE INTERFAZ & \\
\hline TEXTO & \\
\hline $\begin{array}{l}\text { Organizar en párrafos cortos, sin romper los párrafos ni la continuidad de las ideas que se } \\
\text { exponen en ellos. }\end{array}$ & \\
\hline Utilizar hipertexto para dividir información extensa en múltiples páginas. & 5 \\
\hline Marcar bloques de contenido a través de títulos o epígrafes. & 4 \\
\hline Usar mayúsculas para los títulos, encabezados o resaltar textos puntuales. & 4 \\
\hline Evitar subrayados cuando no hay enlaces. & 5 \\
\hline Tipo de letra legible y tamaño adecuado. & 5 \\
\hline Los colores y tipos de letras aportan información por sí mismos. & 4 \\
\hline No presentar ningún error ortográfico. & 4 \\
\hline IMAGEN & \\
\hline Aclarar la información textual. & \\
\hline Su presencia no es superflua. & \\
\hline ANIMACIONES & \\
\hline Las animaciones están justificadas, no se abusa de ellas. & \\
\hline Atraer la atención del usuario para destacar cosas relevantes. & \\
\hline
\end{tabular}

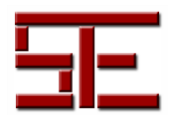

Edwin Insuasty Portilla, Antonio Víctor Martín García y Jesús Insuasti Portilla 


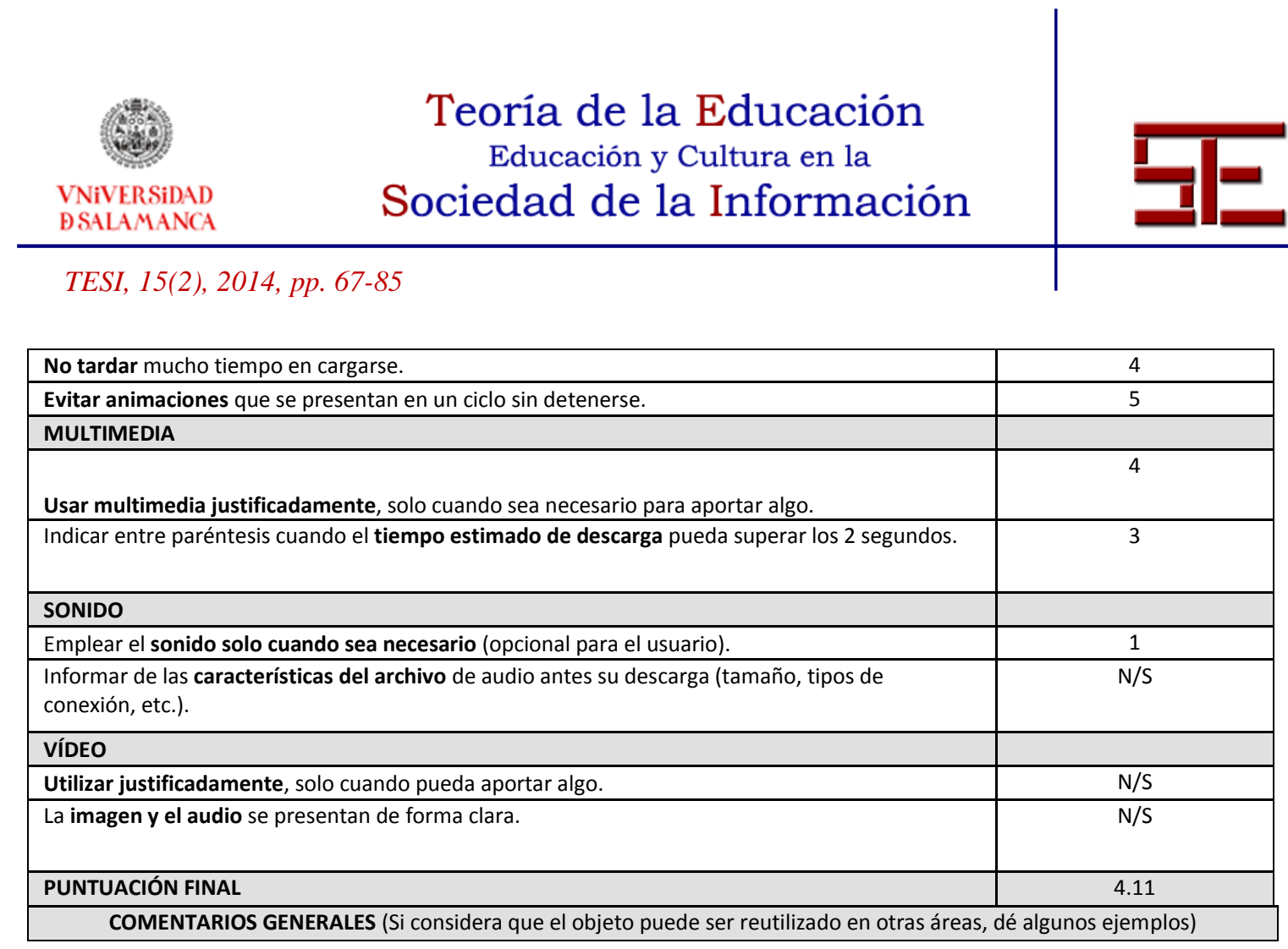

Criterios de Usabilidad (Diseño de Navegación)

\begin{tabular}{|l|c|}
\hline CRITERIOS DE USABILIDAD PARA EVALUAR OBJETOS DE APRENDIZAJE & $\begin{array}{c}\text { N/S No Sabe, } 1 \text { Muy } \\
\text { Deficiente, } 2 \text { Deficiente, } 3 \\
\text { Aceptable, } \\
\text { 4 Alta, } 5 \text { Muy Alta }\end{array}$ \\
\hline DISEÑO DE NAVEGACIÓN & \\
\hline PÁGINA DE INICIO & \\
\hline Aclarar al usuario dónde se encuentra y el objetivo del sitio. & 3 \\
\hline Presentar las principales áreas de contenido del sitio con hipervínculos para acceder a ella. & 4 \\
\hline Si existe pantalla de bienvenida, esta no debe retardar la llegada del usuario a la página de inicio. & 5 \\
\hline NAVEGABILIDAD & \\
\hline Poseer una estructura flexible que permita al usuario controlar su navegación. & \\
\hline Presentar títulos claros indicando nombre o contenido principal. & 5 \\
\hline $\begin{array}{l}\text { La interfaz de navegación muestra todas las alternativas posibles al mismo tiempo, para que los } \\
\text { usuarios puedan escoger su opción. }\end{array}$ & 3 \\
\hline El usuario sabe dónde se encuentra en todo momento. & \\
\hline Las pantallas dedican en gran parte espacio al contenido. & \\
\hline Las páginas deben ser sencillas, no estar recargadas con publicidad, animaciones, etc. & 3 \\
\hline El diseño es consistente en todas las pantallas (tamaños, colores, iconos, tipos de letra, etc.). & 4 \\
\hline PUNTUACIÓN FINAL & 4 \\
\hline
\end{tabular}

Instrumento de evaluación de OAs (Morales Morgado, 2007).

El promedio de los cuatro aspectos evaluados es 3.915.

$800 \begin{gathered}\text { Edwin Insuasty Portilla, Antonio Víctor Martín García } \\ \text { y Jesús Insuasti Portilla }\end{gathered}$




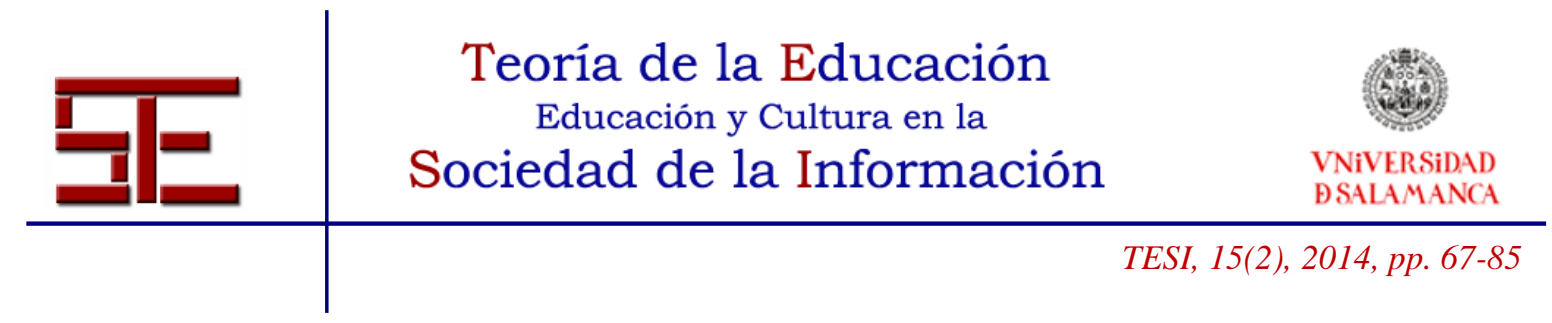

Finalmente se aplicará el instrumento de evaluación usado en la Universidad Autónoma de Aguascalientes. Este instrumento incorpora el concepto de Competencias Desarrolladas. La competencia matemática desarrollada por este Objeto de Aprendizaje es la de Representar, descrita con sus capacidades en lo siguiente: "Representar Incluye las capacidades de:

- Decodificar, interpretar y distinguir entre diferentes tipos de representación de objetos matemáticos y situaciones, así como las interrelaciones entre las distintas representaciones.

- Escoger y relacionar diferentes formas de representación de acuerdo con la situación y el propósito" (Rodríguez; Yordi et al., 2009).

Modelo de evaluación (Ruiz González; Muñoz Arteaga et al., 2007).

\begin{tabular}{|l|l|}
\hline \multicolumn{2}{|l|}{ Formato ECOBA para la evaluación de calidad en los objetos de aprendizaje } \\
\hline Título del OA & Construir la Geometría \\
\hline Temática tratada & Geometría elemental \\
\hline Meta pedagógica & $\begin{array}{l}\text { Ofrecer materiales y propuestas que permiten llevar a cabo prácticas } \\
\text { constructivistas en la enseñanza-aprendizaje del bloque geométrico }\end{array}$ \\
\hline Nivel cognitivo asociado & Comprensión \\
\hline Competencias desarrolladas & $\begin{array}{l}\text { Decodificar, interpretar y distinguir entre diferentes tipos de representación de } \\
\text { objetos matemáticos y situaciones, así como las interrelaciones entre las distintas } \\
\text { representaciones. Escoger y relacionar diferentes formas de representación de } \\
\text { acuerdo con la situación y el propósito }\end{array}$ \\
\hline
\end{tabular}

\begin{tabular}{|c|c|c|c|c|}
\hline Pertinencia y Veracidad de los Contenidos & $\begin{array}{c}\text { Muy } \\
\text { buena } 3 \\
\text { puntos }\end{array}$ & $\begin{array}{l}\text { Buena } 2 \\
\text { puntos }\end{array}$ & $\begin{array}{l}\text { Regular } 1 \\
\text { punto }\end{array}$ & $\begin{array}{l}\text { Mala } 0 \\
\text { puntos }\end{array}$ \\
\hline Presentación del tema a tratar & 3 & & & \\
\hline Especificación de la meta pedagógica & & 2 & & \\
\hline Explicación clara de la temática tratada & & 2 & & \\
\hline \multirow[t]{2}{*}{ Estructuración lógica de los contenidos } & & 2 & & \\
\hline & $\begin{array}{c}\text { Sí } 3 \\
\text { puntos }\end{array}$ & $\begin{array}{l}\text { No } 1 \\
\text { punto }\end{array}$ & & \\
\hline Se proponen ejemplos prácticos y de aplicación & 3 & & & \\
\hline Presenta ejercicios de diagnóstico y evaluación & & 1 & & \\
\hline Se refuerzan los contenidos mediante recursos audiovisuales & 3 & & & \\
\hline $\begin{array}{l}\text { Los contenidos presentan una granularidad que permita su } \\
\text { inclusión dentro de cursos más complejos }\end{array}$ & 3 & & & \\
\hline El OA contiene un metadato con formato estándar & & 1 & & \\
\hline Se presenta la fecha de validez de los contenidos & & 1 & & \\
\hline Los contenidos se consideran vigentes (actualizados) & 3 & & & \\
\hline Se indica el autor/compilador de los contenidos & 3 & & & \\
\hline El autor es considerado capacitado en el tema tratado & 3 & & & \\
\hline Las fuentes de información empleadas son verificables & 3 & & & \\
\hline $\begin{array}{l}\text { Las fuentes de información empleadas son acordes dentro de } \\
\text { la temática tratada }\end{array}$ & 3 & & & \\
\hline
\end{tabular}




\section{\begin{tabular}{ccc} 
Teoría de la Educación \\
$\begin{array}{c}\text { Educación y Cultura en la } \\
\text { VNiVERSADAD } \\
\text { DSALAMANCA }\end{array}$ \\
\hline TESI, 15(2), 2014, pp. 67-85
\end{tabular}}

\begin{tabular}{|c|c|c|c|c|}
\hline Puntaje Total: & 36 & \multicolumn{3}{|c|}{$\begin{array}{l}\text { Puntaje mínimo para considerar } \\
\text { aceptable el OA: } 33\end{array}$} \\
\hline Diseño Estético y Funcional & $\begin{array}{l}\text { Muy } \\
\text { Buena } \\
3 \text { puntos }\end{array}$ & $\begin{array}{c}\text { Buena } 2 \\
\text { puntos }\end{array}$ & $\begin{array}{l}\text { Regular } 1 \\
\text { punto }\end{array}$ & $\begin{array}{c}\text { Mala } \\
0 \text { puntos }\end{array}$ \\
\hline $\begin{array}{l}\text { Pertinencia de los recursos audiovisuales respecto al contenido } \\
\text { textual }\end{array}$ & 3 & & & \\
\hline $\begin{array}{l}\text { Tamaño de los recursos visuales respecto al formato visual del } \\
\text { OA }\end{array}$ & 3 & & & \\
\hline $\begin{array}{l}\text { Distribución de recursos (textuales y audiovisuales) dentro de } \\
\text { los contenidos }\end{array}$ & & 2 & & \\
\hline Legibilidad del texto & & 2 & & \\
\hline Uso de colores para enfatizar la jerarquía temática & & 2 & & \\
\hline $\begin{array}{l}\text { Tamaño del texto respecto a la distribución de contenidos } \\
\text { dentro del OA }\end{array}$ & & 2 & & \\
\hline Rapidez para la carga de recursos audiovisuales & & 2 & & \\
\hline \multirow[t]{2}{*}{ Compatibilidad con distintos navegadores } & 3 & & & \\
\hline & $\begin{array}{l}\text { Sí } 3 \\
\text { puntos }\end{array}$ & $\begin{array}{l}\text { No } 1 \\
\text { punto }\end{array}$ & & \\
\hline Manejo de formatos uniformes dentro del OA & 3 & & & \\
\hline Simetría en la distribución de contenidos y recursos & 3 & & & \\
\hline Los recursos visuales aportan valor agregado al texto & 3 & & & \\
\hline $\begin{array}{l}\text { Se emplean colores para hacer el OA más agradable al } \\
\text { estudiante }\end{array}$ & 3 & & & \\
\hline $\begin{array}{l}\text { El OA cuenta con un sistema de navegación entre contenidos } \\
\text { (Menú o ligas entre contenidos) }\end{array}$ & 3 & & & \\
\hline El OA cuenta con un Metadato estandarizado & & 1 & & \\
\hline $\begin{array}{l}\text { El OA puede ser indexado dentro de un sistema de gestión del } \\
\text { aprendizaje (LMS) }\end{array}$ & 3 & & & \\
\hline Puntaje Total: & 38 & \multicolumn{3}{|c|}{$\begin{array}{l}\text { Puntaje mínimo para considerar } \\
\text { aceptable el OA: } 31\end{array}$} \\
\hline Diseño Instruccional y Aseguramiento de Competencias & $\begin{array}{l}\text { Sí } 3 \\
\text { puntos }\end{array}$ & $\begin{array}{l}\text { No } 1 \\
\text { punto }\end{array}$ & & \\
\hline $\begin{array}{l}\text { Las instrucciones e indicaciones planteadas se plasman de } \\
\text { manera clara }\end{array}$ & 3 & & & \\
\hline $\begin{array}{l}\text { Se encuentran claramente identificadas las habilidades y } \\
\text { capacidades que el estudiante desarrollará mediante la } \\
\text { interacción con el objeto }\end{array}$ & 3 & & & \\
\hline $\begin{array}{l}\text { Se brinda al estudiante el contexto para desarrollar sus propias } \\
\text { conclusiones mediante sus criterios y razonamientos }\end{array}$ & & 1 & & \\
\hline $\begin{array}{l}\text { Las actividades propuestas son acordes al nivel educativo del } \\
\text { contexto para el cual el OA fue creado }\end{array}$ & 3 & & & \\
\hline $\begin{array}{l}\text { Se guía el aprendizaje mediante la estructuración de los } \\
\text { contenidos informativos y/o de las actividades a realizar }\end{array}$ & 3 & & & \\
\hline $\begin{array}{l}\text { Se permite identificar y desarrollar líneas de conocimiento } \\
\text { entre distintos } \mathrm{OA}\end{array}$ & & 1 & & \\
\hline $\begin{array}{l}\text { Los contenidos cubren de manera concreta el tema tratado en } \\
\text { el nivel cognitivo propuesto }\end{array}$ & 3 & & & \\
\hline
\end{tabular}

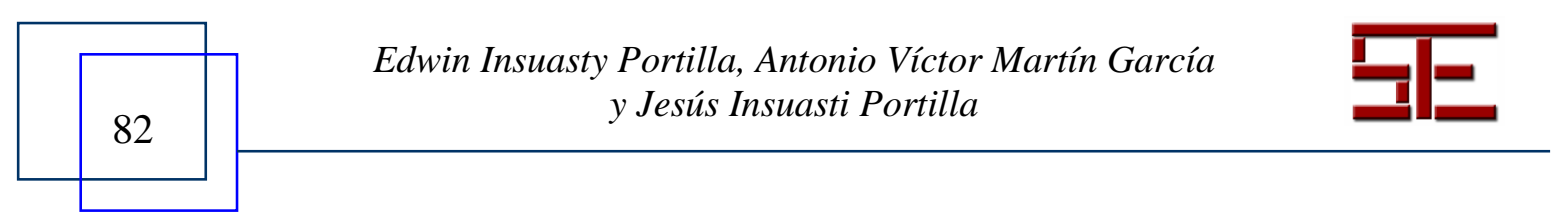




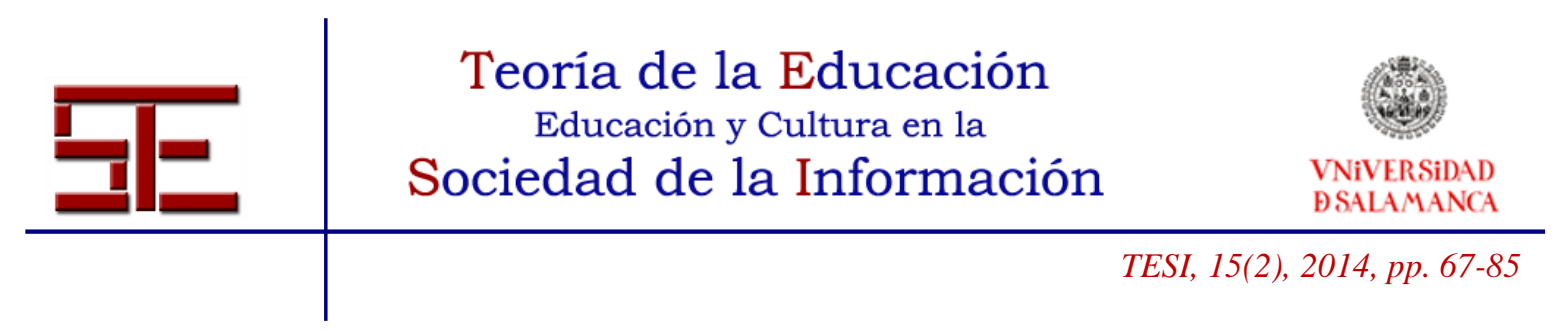

\begin{tabular}{|l|c|c|}
\hline $\begin{array}{l}\text { Las habilidades desarrolladas son acordes con la meta } \\
\text { pedagógica }\end{array}$ & 3 & 3 \\
\hline $\begin{array}{l}\text { La estructuración de contenidos y de actividades son acordes } \\
\text { para el contexto en el cual el OA se implementa }\end{array}$ & 3 & \\
\hline Se fomenta el trabajo individual por parte de los estudiantes & 3 & \\
\hline $\begin{array}{l}\text { Se presentan actividades para una retroalimentación a través } \\
\text { del trabajo colaborativo }\end{array}$ & 27 & Puntaje mínimo para considerar \\
\hline Puntaje Total: & aceptable el OA: 23 \\
\hline
\end{tabular}

\begin{tabular}{|c|c|c|c|}
\hline \multirow[b]{2}{*}{ Nivel de Calidad alcanzado por el OA } & & \multicolumn{2}{|c|}{$\begin{array}{c}\text { Escala para la determinación de } \\
\text { calidad del OA }\end{array}$} \\
\hline & & Mayor de 114 & Excelente \\
\hline & & $105-114$ & Muy buena \\
\hline \multirow{3}{*}{$\begin{array}{l}\text { Puntaje total : Sumando puntajes aprobatorios en las tres } \\
\text { categorías }\end{array}$} & \multirow[t]{3}{*}{101} & $96-105$ & Buena \\
\hline & & $87-96$ & Aceptable \\
\hline & & Menor de 87 & No aceptable \\
\hline
\end{tabular}

\section{4.- CONCLUSIONES}

Estas metodologías de evaluación de OA tienen características semejantes que las llevan a apuntar en una misma dirección, lo que indica que se puede hacer un estudio que permita desarrollar otra metodología a partir de estas tres.

Lo anterior se puede observar en que las tres metodologías de evaluación aplicadas al objeto "Construir la Geometría" proporcionan resultados semejantes que de manera general apuntan a que el OA evaluado tiene una calificación de "Bueno".

El paso siguiente es la catalogación del objeto dentro de un Repositorio y para ello es necesario convertirlo a un estándar admisible, como por ejemplo en un paquete SCORM que permite incorporar el objeto en cualquier sistema e-learning que soporte esta norma como MOODLE.

Cuando se crea un repositorio de Objetos de Aprendizaje tiene que pensarse en la implementación de un mecanismo de evaluación externo que determine la calidad educativa de los objetos de aprendizaje para garantizar a los potenciales usuarios la idoneidad y efectividad de los mismos. Por otra parte, se debe fomentar en la comunidad académica la valoración de diversos objetos que están de manera independiente en la Red para evaluarlos y después de obtener los resultados de dichas valoraciones hacer el contacto con los autores para animarlos a publicar sus objetos en un repositorio, hacerles las sugerencias pertinentes o solicitarles respetuosamente que retiren los objetos de circulación en aras de una educación de mejor calidad.

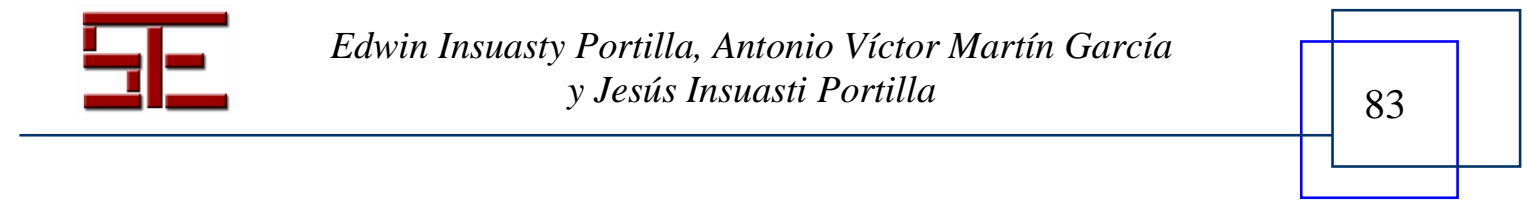




\section{Teoría de la Educación \\ Educación y Cultura en la \\ VNiVERSIDAD \\ DSALAMANCA \\ TESI, 15(2), 2014, pp. 67-85}

\section{5.- BIBLIOGRAFÍA}

Acker, S. R., Pearl, D. K. et al. (2003). Is the Academy Ready for Learning Objects?

En C. M. Gynn \& S. R. Acker, Learning Objects: Contexts \& Connections. Columbus USA: The Ohio State University.

Ferran Ferrer, N. \& Minguillón, J. (2005). Información cualitativa sobre el uso de los objetos de aprendizaje. En II Simposio Pluridisciplinar sobre Diseño, Evaluación y Descripción de Contenidos Educativos Reutilizables (SPDECE). Barcelona, España: UOC.

Friesen, N. (2004). Three Objections to Learning Objects and E-learning Standards. En R. McGreal (Ed.), Online Education Using Learning Objects. London UK: Routledge.

Gibbons, A. S., Nelson, J. et al. (2002). The Nature and Origin of Instructional Objects. En D. Wiley (Ed.), The Instructional Use of Learning Objects. Association for Educational Communications and Technology.

Halm, M. (2003). Beyond the LOM: A New Generation of Specifications. En C. M. Gynn \& S. R. Acker, Learning Objects: Contexts and Connections. Columbus USA: The Ohio State University.

Lightle, K. S. (2003). Using Metadata Standards to Support Interoperability. En C. M. Gynn \& S. R. Acker, Learning Objects: Contexts and Connections. Columbus USA: The Ohio State University.

Morales Morgado, E. M. (2007). Gestión del conocimiento en Sistemas e-learning, basado en objetos de aprendizaje, cualitativa y pedagógicamente definidos. Tesis Doctoral. Departamento de Teoría e Historia de la Educación. Salamanca, España: Universidad de Salamanca.

Morales, E., García, F. J. et al. (2005). Propuesta de Evaluación de Objetos de Aprendizaje. En II Simposio Pluridisciplinar sobre Diseño, Evaluación y Descripción de Contenidos Educativos Reutilizables (SPDECE). Barcelona, España: UOC.

Northrup, P. (2007). Learning Objects for Instruction: Design and Evaluation. London: Information Science Publishing.

Rodríguez, M. L., Yordi, I. et al. (2009). Indicaciones para el logro de competencias geométricas con una visión holística del Álgebra Lineal y la Geometría Analítica en los estudiantes de Arquitectura y de Ingeniería de la Universidad de Camagüey. Revista Iberoamericana de Educación, vol. 49, n. ${ }^{\circ} 4$.

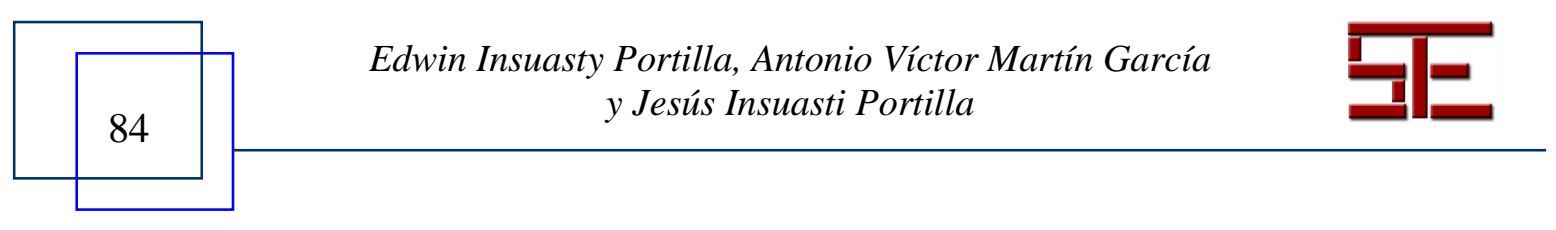




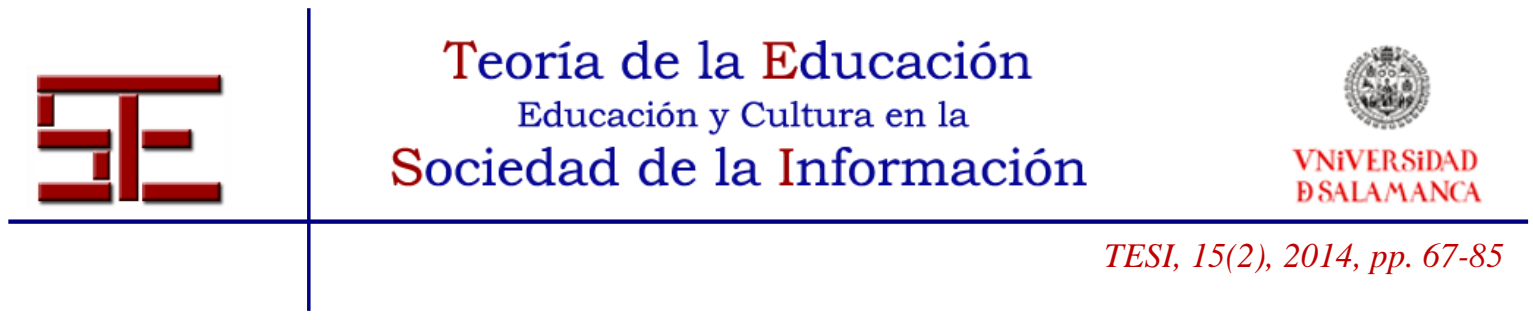

Ruiz González, R. E., Muñoz Arteaga, J. et al. (2007). Evaluación de Objetos de Aprendizaje a través del Aseguramiento de Competencias Educativas. En Virtualeduca Brasil 2007. São José dos Campos, Brasil: Universidade do Vale do Paraiba.

Sanz Rodríguez, J.,. Dodero, J. M. et al. (2008). Evaluación de la Reusabilidad de los Objetos de Aprendizaje. En V Simposio Pluridisciplinar sobre Diseño y Evaluación de Contenidos Educativos Reutilizables. Salamanca, España: Universidad Pontificia de Salamanca.

Vidal, C. L., Segura, A. A. et al. (2008). Calidad en objetos de aprendizaje. En V Simposio Pluridisciplinar sobre Diseño y Evaluación de Contenidos Educativos Reutilizables. Salamanca, España: Universidad Pontificia de Salamanca.

Wiley, D. (2002). Connecting learning objects to instructional design theory: A definition, a metaphor, and a taxonomy. En D. Wiley (Ed.), The Instructional Use of Learning Objects. Association for Educational Communications and Technology.

Wiley, D. (2003). The Coming Collision between Automated Instruction and Social constructivism. En C. M. Gynn \& S. R. Acker, Learning Objects: Contexts \& Connections. Columbus, USA: The Ohio State University.

Williams, D. D. (2002). Evaluation of learning objects and instruction using learning objects. En D. Wiley (Ed.), The Instructional Use of Learning Objects. Association for Educational Communications and Technology.

Para citar el presente artículo puede utilizar la siguiente referencia:

Insuasty Portilla, E., Martín García, A. V. y Insuasti Portilla, J. (2014). Comparación de tres metodologías de evaluación de objetos de aprendizaje virtuales. Revista Teoría de la Educación: Educación y Cultura en la Sociedad de la Información. 15(2), 67-85 [Fecha de consulta: $\mathrm{dd} / \mathrm{mm} / \mathrm{aaaa}]$.

http://campus.usal.es/ revistas_trabajo/index.php/revistatesi/article/view/11887

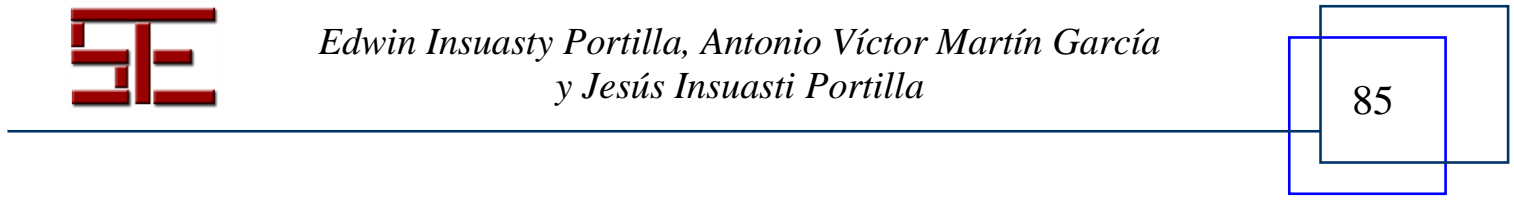

